\title{
Black Caregivers' Perspectives on Racism in ASD Services: Towards Culturally Responsive ABA Practice
}

\author{
Marija Čolić, ${ }^{1}$ Sho Araiba, ${ }^{2}$ Temple S. Lovelace, ${ }^{3}$ and Sarah Dababnah ${ }^{4}$ \\ ${ }^{1}$ University of Hawaii at Manoa, Special Education Department \\ ${ }^{2}$ Positive Behavior Support Corporation, Hawaii, USA \\ ${ }^{3}$ Duquesne University, Department of Counseling, Psychology, and Special Education \\ ${ }^{4}$ University of Maryland, School of Social Work
}

\begin{abstract}
Author Note
Marija Čolić https://orcid.org/0000-0002-9700-458X

Temple S. Lovelace https://orcid.org/0000-0002-1467-1038

Sarah Dababnah https://orcid.org/0000-0001-8298-1639

No external funding was received for the research reported in the paper.

The authors declare that they have no conflict of interest.

Correspondence concerning this article should be addressed to Marija Čolić, University of Hawaii at Manoa, Special Education Department, 1776 University Avenue Wist Hall 120 Honolulu, Hawaii 96822. Email: colicmarija@outlook.com
\end{abstract}


TOWARDS CULTURALLY RESPONSIVE ABA PRACTICE

\begin{abstract}
Significant racial and ethnic disparities in healthcare and service access exist. In the present paper, we reviewed qualitative studies investigating racism-related experiences of Black caregivers of children with Autism Spectrum Disorder (ASD) in the U.S. healthcare system. Specifically, we examined institutional racism (i.e., systemic racism) and individual racism of Black families when they seek diagnoses and services for their children with ASD. Additionally, we summarized culturally responsive and context-specific practice guidelines to work collaboratively with Black caregivers of children with ASD for Applied Behavior Analysis practitioners.

Keywords: autism, applied behavior analysis, Black caregivers, racial discrimination, systemic racism
\end{abstract}


TOWARDS CULTURALLY RESPONSIVE ABA PRACTICE

This manuscript is being published on an expedited basis, as part of a series of emergency publications designed to help practitioners of applied behavior analysis take immediate action to address police brutality and systemic racism. The journal would like to especially thank Associate Editor, Dr. Kaston Anderson-Carpenter. Additionally the journal extends thanks to Drs. Shameka McCammon and Marlesha Bell for their insightful and expeditious reviews of this manuscript. The views and strategies suggested by the articles in this series do not represent the positions of the Association for Behavior Analysis, International or Springer Nature.

Guest Editor, Denisha Gingles 


\section{Black Caregivers' Perspectives on Racism in ASD Services: Towards Culturally}

\section{Responsive ABA Practice}

Research has shown that there are disparities in healthcare and access to services between Black $^{1}$ and white ${ }^{1}$ families in several different domains such as diagnosis, access to care, and the quality of care in the U.S. (Liptak et al., 2008; Magaña et al., 2012; Magaña et al., 2015; Mandell et al., 2002; Montes \& Halterman, 2011). Many studies have pointed out Black children with Autism Spectrum Disorder (ASD) are diagnosed later, more often misdiagnosed with a conductrelated or adjustment disorder, and more likely to be not diagnosed at all compared to their white counterparts (Mandell et al., 2002; Mandell et al., 2009; Williams et al., 2020; but see Emerson et al., 2016, for different results). Furthermore, Black children were less likely to receive early intervention programs (Feinberg et al., 2011) and overall service access than white children (Liptak et al., 2008).

Researchers have attributed racial disparities to many factors such as provider-caregiver interactions, accessibility to services, and geographical differences (Mandell et al., 2002; Williams et al., 2020), which are rooted in the U.S.'s long history of racial segregation and discrimination. Disparities in educational opportunities in communities of color have also contributed to few Black behavioral and mental health professionals (Williams et al., 2020), which often means Black caregivers are interacting with non-Black providers. Racism has a clear impact on caregivers, as previous research showed that racial discrimination among Black

\footnotetext{
${ }^{1}$ In the fields of social sciences, it is a common practice to use the term "Black and African American" to refer to people of this racial/ethnic group, as some members preferred to be called Black and others African American (Sigelman et al., 2005). From here on, we use Black to refer to people of African descent, but we acknowledge the diversity within this community, including of preferences in terms (e.g., African American, Black, Afro American, Black American, see Benkert et al., 2006; Sigelman et al., 2005). Likewise, we use the term white to refer to a group of people often referred to as white American, Caucasian, and European American. White is lowercased as suggested by the Associated Press (Daniszewski, 2020, July 20).
} 
TOWARDS CULTURALLY RESPONSIVE ABA PRACTICE

mothers is a contributing factor of depressive symptoms (McNeil et al., 2014; Odom \& VernonFeagans, 2010), a concerning finding that calls for a family-centered, culturally responsive practice approach for Black children with ASD.

\section{Toward Culturally Responsive and Context-Specific ABA Practice}

In the present paper, we examined studies in the social science and education literature on Black families' experiences with racism in the U.S. healthcare system (Burkett et al., 2015; Dababnah et al., 2018; Gourdine et al., 2011; Lovelace et al., 2018; Pearson \& Meadan, 2018; Solomon \& Lawlor, 2013; Solomon et al., 2015). The main method for data collection used in these studies is qualitative caregiver interviews. Qualitative interviews are not commonly used by behavior analysts, however, these methods can be valuable sources of information when one investigates the conditions in which socially significant behavior is observed in a given verbal community. For these studies, Black caregivers' reports of racist experiences in their interactions with the U.S. healthcare system are critical concerns that the behavior analytic community need to consider. By examining these studies, we wish to contribute to behavior analysts' ability to widen their approaches to the investigation of the social validity of their practice when working with Black families (see Robertson, 2016).

There has been much discussion on culturally responsive practice in Applied Behavior Analysis (ABA; see Special Section on Diversity and Inclusion Issue in Behavior Analysis in Practice). As Brodhead (2019) advocated, culture always matters when implementing ABA services. Such discussion often results in a broad and general set of themes and training content that are not context-specific, given its difficulty narrowing down the characteristics of a given culture (see Fong et al., 2016, for discussion). This difficulty partly stems from a behavior analyst's practice to operationalize each concept; and indeed, it is a daunting task to 
TOWARDS CULTURALLY RESPONSIVE ABA PRACTICE

operationalize each cultural practice. Our approach provides an alternative method to develop context-specific practices. That is, we start by looking at the occurrences of verbal behavior of a group member related to racial discrimination in a particular context and analyze what environmental arrangements cause such verbal instances. Then, we can develop guidelines to prevent these issues in a specific context.

Our current paper focuses on Black caregivers of children with ASD in the context of the U.S. healthcare system. Given racism may not be observable by simply looking at healthcare policies and regulations, we must turn to the lived experiences of people of color interacting with these institutions to examine their reports of racial discrimination. In the following sections, we briefly define our working definitions of racism. Then, we outline findings from several qualitative studies on how different types of racism can manifest themselves in the lives of families raising Black children with ASD (Burkett et al., 2015; Dababnah et al., 2018; Gourdine et al., 2011; Lovelace et al., 2018; Pearson \& Meadan, 2018; Solomon \& Lawlor, 2013; Solomon et al., 2015). Finally, after we explore the service system from Black caregivers' perspectives and point to situations where context specific issues might arise between Black families and ABA practitioners, we provide recommendations for ABA practitioners to prevent and address instances of racism. We believe that our paper continues the important conversations of highlighting the need for culturally responsive and context-specific practice in ABA.

\section{Why is Understanding Race and Racism Important?}

Prior to incorporating recommendations for practice, it is critically important to understand the definition of race and racism. Borrowing from the social sciences, we are using conceptual definitions, a set of abstract characteristics associated with a given concept/term (Timasheff, 1947), which is important when investigating race. Race is a "socially constructed 
TOWARDS CULTURALLY RESPONSIVE ABA PRACTICE

belief that the human race can be divided into biologically discrete and exclusive groups" that are based upon physical, cultural, or social classifications (Golash-Boza, 2016, p. 130; see also Matsuda et al., 2020, for their attempt to operationally define racism). As a related construct, racism represents ideologies and their resultant practices that introduce racial inferiority and superiority (Golash-Boza, 2016). In a social system, these practices can be enacted through individuals and institutions. Individual racism can be both intentional and unintentional, and could manifest as disrespect, bigotry, devaluation, and dehumanization (Golash-Boza, 2016; Jones, 2000). Institutional racism (i.e., systemic racism) is manifested as unequal access to both materials and societal resources (e.g., education, employment, advocacy, infrastructure) for people of color, which includes access to quality medical services (see Golash-Boza, 2016, for review; Jones, 2000). Thus, it is important to investigate not only how racism impacts the individuals that we support, but manifests itself within all social institutions, such as education and healthcare.

\section{Racism Manifested Through Institutions}

"The White community may have access to more information than the Black community" (Burkett et al., 2015, p. 3249).

Institutional racism manifested itself in several ways in our review of the literature of caregivers raising children with ASD. Specifically, we noted instances of racialized differences in access to resources (e.g., information, services), provider interactions (e.g., service rejections, few Black therapists), and clinical research, all rooted in systemic inequities. These findings situate the difficulties Black caregivers of children with ASD face in a much larger historical and societal context, rather than attribute them to a lack of personal effort. 
TOWARDS CULTURALLY RESPONSIVE ABA PRACTICE

In the quotation above, a Black caregiver of children with ASD reported racial differences in available information. This theme of differential access to information based on race rose often in the studies we reviewed. For example, studies reported some parents shared that, due to the lack of educational information about ASD, they had to learn about ASD by themselves, very often through the Internet (Burkett et al., 2015; Lovelace et al., 2018). This type of challenge points to the dearth of educational programs for Black parents in their community. Without systemic and provider supports, Black caregivers needed to educate themselves on ASD.

Systemic challenges persisted for Black families in the ASD service system. Black caregivers reported multiple service rejections for reasons unknown to them (Lovelace et al., 2018). Pearson and Meadan (2018) noted that nine out of 11 Black mothers in their study had to advocate for their children with ASD to access services. Similarly, Solomon and Lawlor (2013), while interviewing Black mothers of children with ASD in Los Angeles, noted that racial discrimination emerged as a frequent theme. That is, mothers reported that they and their children were mistreated by "institutions authorizing autism-related services" (p. 107). Furthermore, mothers in this study shared that their children were denied services and parents were "looked down" upon, and mothers noted that these negative experiences are mostly because of racial discrimination (p. 107). Other studies found that Black families experienced a lack of or a delay in services because of ineffective community institutions. For example, "nice programs" were far from their homes and inaccessible without a car (Lovelace et al., 2018, p. 12). Likewise, Pearson and Meadan (2018) reported that Black caregivers in rural areas shared they could not access ABA services as the centers were too far and there were no in-home sessions because of the lack of nearby therapists and clinicians. Additionally, some Black caregivers noted a 
TOWARDS CULTURALLY RESPONSIVE ABA PRACTICE

differential quality of treatment based on insurance type, and that children with public insurance

did not receive equally good-quality healthcare service compared to private insurance (Burkett et al., 2015). Williams et al. (2020) found that when Black families sought services, they would face multiple difficulties in the healthcare system, and as a result, many of them would not ultimately receive services.

In addition to accessibility issues because of structural inequities, it is important to reflect on the lack of clinical research that included the Black population (see Brodhead et al., 2014, for review of participants' cultural background). Davenport et al. (2018), for example, conducted a systematic review aiming at examining the sample diversity in research on social skills interventions for children with ASD and whether the studies culturally adapted their interventions based on the sample characteristics. Their analysis found that in 79 U.S. studies, only $6.2 \%$ included Black children in the sample (compared to $72.1 \%$ white children), and no studies adapted their interventions for Black participants. Thus, when Black families seek support through the healthcare system, there is a small chance that they will find a treatment that is tailored specifically to Black children and their families, despite the clear value of cultural adaptations (see Robertson, 2016, for social validity of parent-implemented behavior intervention for Black children with ASD).

\section{Racism Manifested through Interactions with Healthcare Providers}

In addition to fighting against and eliminating systemic racism, ABA practitioners need to be cognizant of the ways in which individuals in the healthcare system can display discriminatory activities. The assumptions, stereotypes, and beliefs about clients, conscious or not, can impact a person's ability to deliver relevant and effective services (Garb, 1997). For example, a service provider might have an assumption of a Black client based on race (prejudice) 
TOWARDS CULTURALLY RESPONSIVE ABA PRACTICE

and act upon that assumption (discrimination). One study found a Black mother of a child with ASD experienced racial discrimination in her interactions with her child's doctor, saying, "You assume because I'm Black. . that I might not be as smart. . There are assumptions like. . I'm just this person that has multiple kids. This is a Black woman. If you see my three kids, you assume I don't have a husband" (Dababnah et al., 2018, p. 329). As this quote demonstrates, discrimination that caregivers report does not necessarily have to be explicit to be harmful. Rather, service providers may unconsciously make assumptions and related comments due to implicit bias (Hagiwara et al., 2020).

Disrespectful interactions with healthcare professionals led some Black parents to believe that unfair treatment was due at least partly to their race (Burkett et al., 2015; Dababnah et al., 2018; Lovelace et al., 2018; Pearson \& Meadan, 2018; Solomon \& Lawlor, 2013). For example, one mother said:

Like I've said I've had lots of bad experiences. I can't think of anything else to put it on other than my color... and you know we're in [primarily White neighborhood]....You hate to say it's because of your color...I try to look at everything else and then when everything else doesn't all line up then you have to say maybe it was my color. Maybe it was because I was Black. Maybe it was because I didn't come in... talking in a certain way... maybe they had a different expectation of me and when they saw I had expectations for my child, maybe they didn't want to fulfill that. You don't want to think that way but you have to look at that. It's too blatant for you not to." (Lovelace et al., 2018; p. 7).

In this section, we discuss Black caregivers' experiences facing racism in two healthcare situations: diagnosis and services. 


\section{Experiencing Racism during the Diagnostic Process}

Lovelace et al. (2018) reported that race plays a significant role for Black mothers of children with ASD who navigate the healthcare system. For example, one Black mother described the challenges many Black caregivers encounter getting an ASD diagnosis for their

children, "I felt like a lot of the psychiatrists and stuff that, like, do you think I'm lying? ... I was ignored or what I was saying was trivial to them, and it was very demeaning." (Lovelace et al., 2018; p. 8). Although a timely diagnosis is crucial in the treatment of ASD symptoms (Harris \& Handleman, 2000), Black parents of children with ASD reported multiple experiences of racism in the diagnostic process, which very often contributed to delayed diagnoses. A delay in diagnosis is not only counterproductive to skill acquisition, but also can contribute to increased risk of challenging behaviors. For instance, Solomon and Lawlor (2013) found a parent of a Black girl with ASD reported that a delayed diagnosis compromised her daughter's safety, as she had severe elopement behavior.

The literature we reviewed provided numerous examples of healthcare providers' disrespectful behavior towards caregivers, including neglecting parents' worries regarding their child's growth, minimizing caregivers' knowledge regarding their children, making negative assumptions about Black caregivers' socioeconomic status, and accusing parents of lying about their child's symptoms (Burkett et al., 2015; Dababnah et al., 2018; Gourdine et al., 2011; Lovelace et al., 2018; Pearson \& Meadan, 2018). For example, one mother of a Black child said, "I remember telling [pediatrician] that [child] is not saying any words. The pediatrician said, 'Well, some kids, it takes some time...' ... I kept going to the pediatrician. 'Something's not right. 
TOWARDS CULTURALLY RESPONSIVE ABA PRACTICE

Why is he not speaking?' and [pediatrician] kept coming up with all these excuses..."

(Dababnah et al., 2018, p. 328). This mother tried to obtain help from a doctor to get her child evaluated, but faced multiple obstacles in the healthcare system to do so. In another example, some healthcare professionals suggested caregivers institutionalize their child, even before asking the parents' opinions or assessing their caregiving skills and resources (Gourdine et al., 2011).

Mandell et al. (2002) found that Black parents on average made three times more visits to the doctors to obtain ASD diagnosis than white counterparts. Similarly, Black parents needed to make multiple visits to medical offices in order to receive a referral for a diagnostic evaluation (Dababnah et al., 2018; Pearson \& Meadan, 2018). Due to the prolonged negotiation with healthcare providers, in some cases, these challenges led some parents to seek support through advocacy (Pearson \& Meadan, 2018) or request intervention directly "without their provider's referral" (Dababnah et al., 2018, p. 328). The protracted struggles for referrals, as well as healthcare providers' attitudes towards Black families were characterized by Black caregivers as frustrating, discriminatory, insensitive, and demeaning (Dababnah et al., 2018; Lovelace et al., 2018; Pearson \& Meadan, 2018; Solomon \& Lawlor, 2013).

\section{Experiencing Racism While Seeking and Accessing Services}

Even after Black families obtained their child's ASD diagnosis, the interactions with healthcare providers were still challenging. Families experienced different discriminatory treatments such as a wraparound service specialist's avoidance of integrated community outings in a Black client's neighborhood (Lovelace et al., 2018). The mother attributed the specialist's repeated denials of the community outings to the specialist's belief that the family's 
TOWARDS CULTURALLY RESPONSIVE ABA PRACTICE

neighborhood was not safe for community programs, although the family lived in a mainly white neighborhood. The mother stated, "I didn't live right in the shoot 'em up central." (p. 7).

Another example was a Black mother's report that her doctor wrongly judged her by saying, “'Well, you can't do this with Medicaid.' I said, 'I don't even have Medicaid, I have private insurance."” (Dababnah et al., 2018, p. 329).

Even when their children received services, some caregivers reported that healthcare providers did not take into consideration parental knowledge regarding their children and their opinions regarding treatment options (Burkett et al., 2015; Lovelace et al., 2018). While parents desired a good relationship with healthcare professionals, perceptions about how professionals evaluated their ability to implement effective treatment at home overshadowed how caregivers interacted with them (Burkett et al., 2015).

Caregivers' previous experiences of explicit and implicit racism often contributed to parental behavior change; in that they became vigilant in interactions with healthcare providers. For example, a Black mother withdrew her child with ASD from a service because she felt her child was not welcomed, and the mother did not want to expose her child to negative experiences (Lovelace et al., 2018). When they did bring their children to professional care, caregivers reported being watchful of their children's interactions with the professionals, because they did not fully trust healthcare professionals (Burkett et al., 2015). Solomon et al. (2015) reported that the mother of a Black child with ASD was vigilant in healthcare settings because of the potential "intersection of race and disability (i.e., the direct and implicit ways in which race can exacerbate potential impediments to her son's care)" (p. 550). Therefore, when the mother was asked to check off whether her son was Black while filling out the form during the doctor visit, she decided to write "human" instead. Furthermore, Lovelace et al. (2018) said when seeking 
TOWARDS CULTURALLY RESPONSIVE ABA PRACTICE

services, a mother sometimes modified her accent over the phone, as she feared rejection from professional services if they realized she was Black. The authors wrote:

Often parents have to resort to code-switching or speaking in a manner that can mask another's perceptions as to their race or ethnicity. While on a phone call, Latisha said that on the phone she can speak 'prim and proper' and doesn't always 'sound' African American, but the person figured it out and didn't let her son in (Lovelace et al., 2018, p. $8)$.

\section{Recommendations for Culturally Responsive and Context-Specific ABA practice}

Black families of children with ASD face multiple difficulties navigating the healthcare system. It is important that $\mathrm{ABA}$ practitioners recognize the potential barriers Black families may have overcome to arrive at the point where they request ABA service. Thus, ABA practitioners should embrace the perspectives and values shared by Black families to not only facilitate the effectiveness of their interventions, but also fight against racial discrimination and promote equity $^{2}$ in healthcare services (Burkett et al., 2015; Dababnah et al., 2018; Lovelace et al., 2018; Pearson \& Meadan, 2018).

Although the barriers that Black families of children with ASD face may not always be tied to their race (e.g., white and other racial/ethnic groups also reported certain challenges; see Sansosti et al., 2012), the frequency with which racial discrimination is experienced by Black individuals and their caregivers is of great concern (e.g., Burkett et al., 2015; Dababnah et al., 2018; Lovelace et al., 2018). Black families have reported that having trusting relationships with healthcare professionals is very important for them. Their reports provide insights into the values

\footnotetext{
${ }^{2}$ In the context of racial justice within the healthcare system, equality refers to providing standardized service to people of all ethics backgrounds, whereas equity refers to ensuring culturally responsive service.
} 
TOWARDS CULTURALLY RESPONSIVE ABA PRACTICE

and competencies that healthcare professionals need to develop to build a good relationship with Black families of children with ASD (Burkett et al., 2015; Pearson \& Meadan, 2018).

This issue is timely for behavior analysts. The demand for ABA services in the U.S. has been increasing constantly over the years "with a 1,942\% increase from 2010 to 2018 and a $127 \%$ increase from 2017 to 2018 " (BACB, 2019, p. 2). With the increasing demands, we would expect that more ABA practitioners will provide services to culturally diverse populations. Therefore, training on cultural diversity and cultural humility is needed for a successful ABA services provision (see Dennison et al., 2019; Fong et al., 2016; Fong \& Tanaka, 2013; Wright, 2019, for discussion on cultural diversity and cultural humility). However, recent surveys with Behavior Analyst Certification Board (BACB) certified practitioners show that graduate training, fieldwork, and supervision do not provide adequate education on multiculturalism and diversity (Beaulieu et al., 2019; Conners et al., 2019) and that there are very little opportunities for continued education on these topics (Beaulieu et al., 2019). In the following sections, we discuss implications and recommendations for ABA practitioners to fight against racism in the U.S. healthcare system.

\section{Combating Institutional Racism}

Although the BACB recently began providing public information regarding the racial and ethnic backgrounds of its certified ABA practitioners, the lack of racially diverse representation is apparent from the shortage of multilingual and multicultural personnel and training programs (Dennison et al., 2019). The behavior analytic field can increase the diversity of ABA professionals through targeted opportunities for Black professionals to choose future careers in ABA and the support of individuals through scholarships, mentorships, and advocacy (Fong et al., 2016). Additionally, there has been an increasing effort to empower the Black community 
TOWARDS CULTURALLY RESPONSIVE ABA PRACTICE

within the ABA field through the creation of networks, such as Black Applied Behavior Analysts, Inc. This, combined with a more inclusive and responsive behavior analytic field, will more likely help mitigate institutional racism.

Furthermore, our field can help increase Black communities' access to culturally relevant information on ASD, culturally responsive ABA approaches, assessments and interventions of ASD, parent training on policy and advocacy concerns that are important for racially diverse communities. In addition, the field can be more forward-thinking, combating the lack of practitioners and clinical options in certain rural communities. Although the COVID-19 pandemic has increased the availability of telehealth options for caregivers and their children (see Unholz-Bowden et al., 2020, for a review of telehealth effectiveness), continued efforts to advocate for underserved populations is needed to alleviate some of the impacts of institutional racism.

\section{Combating Provider Racial Bias}

In some cases, ABA practitioners might not be aware of their own biases towards Black communities and may unintentionally engage in racial discrimination while providing services. Based on the summary of qualitative studies above, we identified four main areas where ABA practitioners should be especially sensitive in order to diminish racism manifested via interactions: 1) build knowledge about Black cultural values, 2) show care and strengthen partnerships, 3) create culturally relevant interventions, and 4) be aware of one's own prejudice and cultural values.

\section{Build Knowledge about Black Cultural Values}

ABA practitioners should make meaningful efforts to understand the cultural values of each family they serve, although one should not have an a priori assumption that each family 
TOWARDS CULTURALLY RESPONSIVE ABA PRACTICE

adopts the same cultural values. We learn about the values that a particular family endorses during initial and ongoing assessments and services provision. Although there is considerable variation in cultural values within Black communities, we highlight two major cultural themes from the literature: 1) respect and 2) awareness of Black families' attitudes toward ASD.

Respect. Respect is an important value in Black community and it has been passed down in families over generations (Burkett et al., 2017). A child that "behaved" showed respect for others and reflected the discipline practices of their parents. As one Black father described, "You did not disrespect your mom or dad... even if your father wasn't in your life." (Burkett et al., 2017, p. 498). They view parenting as an important way to teach respect to their children and would employ traditional and nontraditional discipline practices such as directives or mimicking the disruptive behavior of their children. Black parents tend to expect their children with ASD to live up to the same standards as that of their typically developing siblings (Burkett et al., 2015; Burkett et al., 2017). Understanding these values may explain some Black families' preferences for certain treatment options and increase their adherence to the treatment plans. Pearson et al. (2018) proposed that speech-language pathologists (SLPs) should value Black families' beliefs and the role of respect in their lives without judgment. This can also inform how ABA practitioners should approach discussions of treatment procedures with Black caregivers before they are implemented.

Awareness of Black Families' Attitudes Toward ASD. The impacts of institutional and individualized racism have affected how Black families view ASD. Previous studies have highlighted some within the Black community have navigated ASD experience through not believing an ASD diagnosis, associating shame with having a child with ASD, or not immediately agreeing that a child needs an intervention (Dababnah et al., 2018; Lovelace et al., 
TOWARDS CULTURALLY RESPONSIVE ABA PRACTICE

2018; Pearson \& Meadan, 2018). ABA practitioners have to be aware of the impact of social and cultural systems and their influence on Black families' engagement with ASD. For instance, one Black mother suggested that professionals should carefully discuss ASD within the family's broader sociocultural context and build a relationship with the parents before providing services or referring them to other professionals (Pearson \& Meadan, 2018). Furthermore, one Black mother described,

"I think that putting more knowledge out there specifically geared towards African Americans [is important]. Examples of African Americans that have children on the spectrum that are getting services, that are actually benefiting in some way, and encouraging them to be more involved in their child's services, and their child's education as well, it would really, really, really, really, really, help." (Lovelace et al., 2018, p. 12).

\section{Show Care and Strengthen Partnerships}

Respect within the family-professional relationship is very important (Burkett et al., 2015; Lovelace et al., 2018). To better understand how to show care and build a successful parent-professional partnership, we identify two areas: 1) listen to what Black caregivers have to say, and 2) explain service provision, timelines, and objectives transparently.

Listen to What Black Caregivers Have to Say. Black caregivers shared that they need professionals who listen to what they have to say regarding their child and their concerns (Burkett et al., 2015; Lovelace et al., 2018; Pearson \& Meadan, 2018). As presented earlier, professionals have disregarded Black parents' concerns in various healthcare settings (e.g., diagnostics, service planning). Caregivers also reported their children's diagnoses were delayed because their primary healthcare providers did not acknowledge early parental concerns 
TOWARDS CULTURALLY RESPONSIVE ABA PRACTICE

(Dababnah et al., 2018). Thus, it is crucial to listen to Black parents, not only to establish a respectful relationship, but also to facilitate effective screening and intervention processes.

A part of developing a respectful therapeutic relationship with Black families is to include them when developing a service plan for their children. Dennison et al. (2019) discussed the importance of social validity in ABA when working with families from diverse backgrounds. Listening to and acknowledging families' values about treatment outcomes should be important for quality ABA services. As one Black mother of a child with ASD reflected, “I don't really go too much by the blueprint as they say. I try to adapt the blueprint to what I have." (Burkett et al., 2015, p. 3249). Therefore, without parental opinions and feedback, ABA practitioners will be uncertain if a given intervention is relevant for a family and if they will adhere to given recommendations.

Another way to listen to Black families is to examine parents' beliefs about the child's diagnosis, their expectations about treatment, and their knowledge regarding different treatment options systematically (see Mandell \& Novak, 2005, for useful questions to ask). Similarly, La Roche et al. (2018) suggested the Cultural Formulation Interview could also be a beneficial clinical tool to understand cultural factors from parental perspectives related to ASD diagnosis and treatment. Moreover, from a functional contextualism approach, Taylor et al. (2019) developed the training curricula that could equip ABA practitioners with techniques of compassionate care. Their training curricula focus on "active listening, collaborating with caregivers, understanding a family's culture, being kind, asking open-ended questions, avoiding technical jargon, and caring for the entire family" (p. 660).

Explain Service Provision, Timelines, and Objectives Transparently. ABA practitioners should fully explain service provision, service timelines, and service objectives as 
TOWARDS CULTURALLY RESPONSIVE ABA PRACTICE

clearly as possible. Transparency in service provision is an important factor in any families' participation in services. A lack of transparency can impact adherence and continued participation in behavioral health services, and for Black families who experience an increased level of discrimination, service provision must be described in clear, relevant ways (CarpenterSong, 2010, as cited in Williams et al., 2020). Williams et al. (2020) further suggested that transparency between the service providers and the Black clients will decrease mistrust and facilitate collaboration, which is particularly important in order to increase treatment effectiveness and generalization.

\section{Create Culturally Relevant Interventions}

ABA practitioners should adapt and modify their treatment goals to increase cultural relevance. One of the biggest mistakes that providers can make is thinking that there is a singular path to supporting Black families. Working with each family to co-create target goals that reflect the family's value system improves the cultural relevance of treatment recommendations (Kauffman et al., 2008). By including families in treatment design, ABA practitioners will not only understand their preferences for specific goals, but also how a families' experiences shape why certain decisions are made for their children (Burkett et al., 2015).

Creating culturally relevant assessment and intervention materials is also important. For example, Blake et al. (2017) adapted parent-mediated social communication interventions by modifying a picture of a father and a child to that of a mother and a child and changing the women's dresses in the pictures to reflect the clients' cultural norms. In another example, Pearson et al. (2018) discussed how SLPs modified their communication devices such as Picture Exchange Communication System (PECS) and a speech-generating device to adapt to the racial background of families. Specifically, a therapist can alter a picture of a person in PECS to have 
TOWARDS CULTURALLY RESPONSIVE ABA PRACTICE

the same skin tone as that of a Black student or make the generated voice of the speechgenerating device to match with a Black student's family member's voice (dialect, tone, inflection, semantics and other factors). Pearson et al. (2018) also suggested using social stories to teach culturally different expectations that a Black student might encounter between home and school, such as the loudness of a voice or an acceptable level of body movements.

Pearson et al. (2018) also provided valuable suggestions for skill assessments. They argued that SLPs should exercise caution when evaluating Black students' language, and this recommendation is also critical for behavior analysts. Incorporating a Black linguistic consciousness (Baker-Bell, 2020) will help analysts in the evaluation of a Black students' speech patterns and allow them to understand the characteristic verb markings that are incorporated in Black language. As ABA professionals become more mindful of these characteristics when assessing their clients' behavioral repertoire, they will improve effectiveness and social appropriateness of interventions and assessments.

\section{Be Aware of One's Own Prejudice and Cultural Values}

For all professionals, it is important to recognize that no one is free of prejudice. Many researchers (Ford \& Airhihenbuwa, 2010; La Roche et al., 2018) thus emphasized that clinicians should understand their own cultures, biases, and values when they provide services to Black clients in order to improve the quality of interactions with them. Similarly, ABA practitioners should be aware of one's own culture and biases when working with clients (Fong \& Tanaka, 2013; Fong et al., 2016; Wright, 2019). Fong et al. (2016) illustrated how preconceived notions can impact a behavior analyst's effectiveness with families of different backgrounds. The authors offered examples of how the analyst should be aware of their own reactions to information that families share especially when it differs from their own cultural norms such as attitudes toward 
TOWARDS CULTURALLY RESPONSIVE ABA PRACTICE

family and gender roles. In line with the recommendations of Fong et al. (2016) to assess cultural bias, we think it is also important that ABA practitioners seek out professional development on implicit bias and how it can be triggered during the course of service (e.g., see more information on Implicit Bias Training, Hagiwara et al., 2020). Other practice-oriented frameworks offer providers on addressing institutional racism and other forms of oppression within their work (e.g., see Shaia, 2019).

\section{Conclusion}

Despite some hints of improvements in the area of early diagnosis and referral to early intervention programs for Black children with ASD (Dababnah et al., 2018), general disparities in the healthcare system between Black and white families still persist in the U.S. Black caregivers continue to face more obstacles navigating the U.S. healthcare system compared to their white counterparts which leads to irreparable disparities in Black and white individuals with autism. Through our review of qualitative studies that reflect the experiences of Black caregivers of children with ASD with healthcare service providers, we have found that Black caregivers face racism at almost all steps in the healthcare system. ABA practitioners and the behavior analytic field must respond to this persistent social justice issue and act to eliminate the barriers Black families face when accessing the healthcare system, so that their children with ASD can receive proper and effective interventions to improve their quality of life without facing racism. By identifying the contexts in which such racial discrimination might occur, we can develop culturally responsive and context-specific practice to prevent and eliminate racism in the healthcare system. 
TOWARDS CULTURALLY RESPONSIVE ABA PRACTICE

\section{References}

Baker-Bell, A. (2020). Linguistic justice: Black language, literacy, identity, and pedagogy.

New York \& Urbana, IL: Routledge \& National Council of Teachers of English.

Beaulieu, L., Addington, J., \& Almeida, D. (2019). Behavior analysts' training and practices regarding cultural diversity: The case for culturally competent care. Behavior Analysis in Practice, 12(3), 557-575. http://doi.org/10.1007/s40617-018-00313-6

Behavior Analyst Certification Board (BCBA). (2019). US employment demand for behavior analysts: 2010 - 2018. Littleton: Author.

Benkert, R., Peters, R. M., Clark, R., \& Keves-Foster, K. (2006). Effects of perceived racism, cultural mistrust and trust in providers on satisfaction with care. Journal of the National Medical Association, 98(9), 1532-1540.

Blake, J. M., Rubenstein, E., Tsai, P. C., Rahman, H., Rieth, S. R., Ali, H., \& Lee, L. C. (2017). Lessons learned while developing, adapting and implementing a pilot parent-mediated behavioural intervention for children with autism spectrum disorder in rural Bangladesh. Autism, 21(5), 611-621. http://doi.org/10.1177/1362361316683890

Brodhead, M. T. (2019). Culture always matters: Some thoughts on Rosenberg and Schwartz. Behavior Analysis in Practice, 12(4), 826-830. http://doi.org/10.1007/s40617-019-003518

Brodhead, M. T., Durán, L., \& Bloom, S. E. (2014). Cultural and linguistic diversity in recent verbal behavior research on individuals with disabilities: A review and implications for research and practice. The Analysis of Verbal Behavior, 30(1), 75-86.

http://doi.org/10.1007/s40616-014-0009-8 
TOWARDS CULTURALLY RESPONSIVE ABA PRACTICE

Burkett, K., Morris, E., Anthony, J., Shambley-Ebron, D., \& Manning-Courtney, P. (2017). Parenting African American children with autism: The influence of respect and faith in mother, father, single-, and two-parent care. Journal of Transcultural Nursing, 28(5), 496-504. http://doi.org/10.1177/1043659616662316

Burkett, K., Morris, E., Manning-Courtney, P., Anthony, J., \& Shambley-Ebron, D. (2015). African American families on autism diagnosis and treatment: The influence of culture. Journal of Autism and Developmental Disorders, 45(10), 3244-3254. http://doi.org/10.1007/s10803-015-2482-X

Conners, B., Johnson, A., Duarte, J., Murriky, R., \& Marks, K. (2019). Future directions of training and fieldwork in diversity issues in applied behavior analysis. Behavior Analysis in Practice, 12(4), 767-776. http://doi.org/10.1007/s40617-019-00349-2

Dababnah, S., Shaia, W. E., Campion, K., \& Nichols, H. M. (2018). "We had to keep pushing": Caregivers' perspectives on autism screening and referral practices of Black children in primary care. Intellectual and Developmental Disabilities, 56(5), 321-336. http://doi.org/10.1352/1934-9556-56.5.321

Davenport, M., Mazurek, M., Brown, A., \& McCollom, E. (2018). A systematic review of cultural considerations and adaptation of social skills interventions for individuals with autism spectrum disorder. Research in Autism Spectrum Disorders, 52, 23-33. http://doi.org/10.1016/j.rasd.2018.05.003

Dennison, A., Lund, E. M., Brodhead, M. T., Mejia, L., Armenta, A., \& Leal, J. (2019). Delivering home-supported applied behavior analysis therapies to culturally and 
TOWARDS CULTURALLY RESPONSIVE ABA PRACTICE

linguistically diverse families. Behavior Analysis in Practice, 12(4), 887-898. http://doi.org/10.1007/s40617-019-00374-1

Emerson, N. D., Morrell, H. E., \& Neece, C. (2016). Predictors of age of diagnosis for children with autism spectrum disorder: The role of a consistent source of medical care, race, and condition severity. Journal of Autism and Developmental Disorders, 46(1), 127-138. http://doi.org/10.1007/s10803-015-2555-X

Feinberg, E., Silverstein, M., Donahue, S., \& Bliss, R. (2011). The impact of race on participation in Part C early intervention services. Journal of Developmental \& Behavioral Pediatrics, 32(4), 284-291. http://doi.org/10.1097/DBP.0b013e3182142fbd

Fong, E. H., Catagnus, R. M., Brodhead, M. T., Quigley, S., \& Field, S. (2016). Developing the cultural awareness skills of behavior analysts. Behavior Analysis in Practice, 9(1), 84-94. http://doi.org/10.1007/s40617-016-0111-6

Fong, E. H., \& Tanaka, S. (2013). Multicultural alliance of behavior analysis standards for cultural competence in behavior analysis. International Journal of Behavioral Consultation and Therapy, 8(2), 17-19. http://doi.org/10.1037/h0100970

Ford, C. L., \& Airhihenbuwa, C. O. (2010). Critical race theory, race equity, and public health: toward antiracism praxis. American Journal of Public Health, 100(S1), S30-S35. http://doi.org/10.2105/AJPH.2009.171058

Garb, H. N. (1997). Race bias, social class bias, and gender bias in clinical judgment. Clinical Psychology: Science and Practice, 4(2), 99-120. http://doi.org/0.1111/j.14682850.1997.tb00104.x 
TOWARDS CULTURALLY RESPONSIVE ABA PRACTICE

Golash-Boza, T. (2016). A critical and comprehensive sociological theory of race and racism. American Sociological Association, 2(2), 129-141. http://doi.org/10.1177/2332649216632242

Gourdine, R. M., Baffour, T. D., \& Teasley, M. (2011). Autism and the African American community. Social Work in Public Health, 26(4), 454-470. http://doi.org/10.1080/19371918.2011.579499

Hagiwara, N., Kron, F. W., Scerbo, M. W., \& Watson, G. S. (2020). A call for grounding implicit bias training in clinical and translational frameworks. Lancet, 395(10234), 1457-1460. http://doi.org/10.1016/S0140-6736(20)30846-1

Harris, S. L., \& Handleman, J. S. (2000). Age and IQ at intake as predictors of placement for young children with autism: A four to six-year follow-up. Journal of Autism and Developmental Disorders, 30, 137-142. http://doi.org/10.1023/A:1005459606120

Jones, C. P. (2000). Levels of racism: a theoretic framework and a gardener's tale. American Journal of Public Health, 90(8), 1212-1215. http://doi.org/10.2105/ajph.90.8.1212

Kauffman, J. M., Conroy, M., Gardner, R., \& Oswald, D. (2008). Cultural sensitivity in the application of behaviour principles to education. Education and Treatment of Children, 31(2), 239-262. http://doi.org/10.1353/etc.0.0019

La Roche, M. J., Bush, H. H., \& D'Angelo, E. (2018). The assessment and treatment of autism spectrum disorder: A cultural examination. Practice Innovations, 3(2), 107-122. http://doi.org/10.1037/pri0000067

Liptak, G. S., Benzoni, L. B., Mruzek, D. W., Nolan, K. W., Thingvoll, M. A., Wade, C. M., \& Fryer, G. E. (2008). Disparities in diagnosis and access to health services for children with autism: data from the National Survey of Children's Health. Journal of 
TOWARDS CULTURALLY RESPONSIVE ABA PRACTICE

Developmental \& Behavioral Pediatrics, 29(3), 152-160.

http://doi.org/10.1097/DBP.0b013e318165c7a0

Lovelace, T. S., Tamayo, S., \& Robertson, R. E. (2018). Experiences of African American mothers of sons with autism spectrum disorder: Lessons for improving service delivery. Education and Training in Autism and Developmental Disabilities, 53(1), 3-16.

Magaña, S., Parish, S. L., Rose, R. A., Timberlake, M., \& Swaine, J. G. (2012). Racial and ethnic disparities in quality of health care among children with autism and other developmental disabilities. Intellectual and Developmental Disabilities, 50(4), 287-299. http://doi.org/10.1352/1934-9556-50.4.287

Magaña, S., Parish, S. L., \& Son, E. (2015). Have racial and ethnic disparities in the quality of health care relationships changed for children with developmental disabilities and ASD? American Journal on Intellectual and Developmental Disabilities, 120(6), 504-513. http://doi.org/10.1352/1944-7558-120.6.504

Mandell, D. S., Listerud, J., Levy, S. E., \& Pinto-Martin, J. A. (2002). Race differences in the age at diagnosis among Medicaid-eligible children with autism. Journal of the American Academy of Child \& Adolescent Psychiatry, 41(12), 1447-1453. http://doi.org/10.1097/00004583-200212000-00016

Mandell, D. S., \& Novak, M. (2005). The role of culture in families' treatment decisions for children with autism spectrum disorders. Mental Retardation and Developmental Disabilities research reviews, 11(2), 110-115. http://doi.org/10.1002/mrdd.20061

Mandell, D. S., Wiggins, L. D., Carpenter, L. A., Daniels, J., DiGuiseppi, C., Durkin, M. S., Giarelli, E., Morrier, M. J., Nicholas, J. C., Pinto-Martin, J. A., Shattuck, P. T., Thomas, K. C., Yeargin-Allsopp, M., \& Kirby, R. S. (2009). Racial/ethnic disparities in the 
TOWARDS CULTURALLY RESPONSIVE ABA PRACTICE

identification of children with autism spectrum disorders. American Journal of Public Health, 99(3), 493-498. http://doi.org/10.2105/AJPH.2007.131243

Matsuda, K., Garcia, Y., Catagnus, R., \& Brandt, J. A. (2020). Can behavior analysis help us understand and reduce racism? A review of the current literature. Behavior Analysis in Practice, 13, 336-347. http://doi.org/10.1007/s40617-020-00411-4

McNeil, S., Harris-McKoy, D., Brantley, C., Fincham, F., \& Beach, S. R. (2014). Middle class African American mothers' depressive symptoms mediate perceived discrimination and reported child externalizing behaviors. Journal of Child and Family Studies, 23(2), 381388. http://doi.org/10.1007/s10826-013-9788-0

Montes, G., \& Halterman, J. S. (2011). White-Black disparities in family-centered care among children with autism in the United States: Evidence from the NS-CSHCN 2005-2006. Academic Pediatrics, 11(4), 297-304. http://doi.org/10.1016/j.acap.2011.02.002

Odom, E. C., Vernon-Feagans, L., \& Family Life Project Key Investigators. (2010). Buffers of racial discrimination: Links with depression among rural African American mothers. Journal of Marriage and Family, 72(2), 346-359. http://doi.org/10.1111/j.17413737.2010.00704.x

Pearson, J. N., Hamilton, M. B., \& Meadan, H. (2018). “We saw our son blossom”. A guide for fostering culturally responsive partnerships to support African American autistic children and their families. Perspectives of the ASHA Special Interest Groups, 3(1), 84-97. http://doi.org/10.1044/persp3.SIG1.84

Pearson, J. N., \& Meadan, H. (2018). African American parents' perceptions of diagnosis and services for children with autism. Education and Training in Autism and Developmental Disabilities, 53(1), 17-32. 
TOWARDS CULTURALLY RESPONSIVE ABA PRACTICE

Robertson, R. E. (2016). Effectiveness and acceptability of parent-implemented behavior interventions for children with autism in three African American families. Education and Training in Autism and Developmental Disabilities, 51(2), 107-121.

Sansosti, F. J., Lavik, K. B., \& Sansosti, J. M. (2012). Family experiences through the autism diagnostic process. Focus on Autism and Other Developmental Disabilities, 27(2), 81-92. http://doi.org/10.1177/1088357612446860

Sigelman, L., Tuch, S. A., \& Martin, J. K. (2005). What's in a name? Preference for "Black" versus “African-American” among Americans of African descent. Public Opinion Quarterly, 69(3), 429-438. http://doi.org/10.1093/poq/nfi026

Shaia, W. E. (2019). SHARP: A framework for addressing the contexts of poverty and oppression during service provision in the United States. Journal of Social Work Values \& Ethics, 16(1), 16-26.

Solomon, O., Angell, A. M., Yin, L., \& Lawlor, M. C. (2015). "You can turn off the light if you'd like": Pediatric health care visits for children with autism as an interactional achievement. Medical Anthropology Quarterly, 29(4), 531-555. http://doi.org/10.1111/maq.12237

Solomon, O., \& Lawlor, M. C. (2013). “And I look down and he is gone”: Narrating autism, elopement and wandering in Los Angeles. Social Science \& Medicine, 94, 106-114. http://doi.org/10.1016/j.socscimed.2013.06.034

Daniszewski, J. (2020, July 20). Why we will lowercase white. AP. https://blog.ap.org/announcements/why-we-will-lowercase-white

Taylor, B. A., LeBlanc, L. A., \& Nosik, M. R. (2019). Compassionate care in behavior analytic treatment: Can outcomes be enhanced by attending to relationships with caregivers?. 
TOWARDS CULTURALLY RESPONSIVE ABA PRACTICE

Behavior Analysis in Practice, 12(3), 654-666. http://doi.org/10.1007/s40617-018-002893

Timasheff, N. S. (1947). Definitions in the social sciences. American Journal of Sociology, 53(3), 201-209. http://doi.org/10.1086/220142

Unholz-Bowden, E., McComas, J. J., McMaster, K. L., Girtler, S. N., Kolb, R. L., \& Shipchandler, A. (2020). Caregiver training via telehealth on behavioral procedures: A systematic review. Journal of Behavioral Education, 29(2), 246-281. http://doi.org/10.1007/s10864-020-09381-7

Williams, M. T., George, J. R., \& Printz, D. M. (2020). Behavioral health service delivery with African Americans. In L. Benuto, F. Gonzalez, Singer J. (Eds), Handbook of cultural factors in behavioral health (pp. 111-129). Springer, Cham. http://doi.org/10.1007/978-3030-32229-8_9

Wright, P. I. (2019). Cultural humility in the practice of applied behavior analysis. Behavior Analysis in Practice, 12(4), 805-809. http://doi.org/10.1007/s40617-019-00343-8 